\title{
Análise comparativa do maior impacto no orçamento extraído pelo método convencional e pela tecnologia BIM
}

\section{Comparative analysis of the greatest impact on the budget extracted by the conventional method and by BIM technology}

\author{
Gustavo Macedo Miranda ${ }^{1 *}$, Danielle Meireles de Oliveira ${ }^{1}$, Marys Lene Braga \\ Almeida $^{1}$, Sidnea Eliane Campos Ribeiro ${ }^{1}$, Carmen Couto Ribeiro ${ }^{1}$
}

\begin{abstract}
RESUMO
A tecnologia Building Information Modelling (BIM) apresenta-se como uma ferramenta que permite conexão e integração de uma maneira não antes conhecida na construção civil, possibilitando que as disciplinas dos projetos sejam analisadas de forma integrada e viabilizando melhorias instantâneas. Neste estudo, foi realizada uma análise comparativa dos quantitativos obtidos via método convencional e via tecnologia BIM a fim de identificar as divergências entre as ferramentas e seu impacto no orçamento. Com esse objetivo, um empreendimento de pequeno porte foi modelado no software Revit e os quantitativos obtidos foram confrontados com os extraídos via ferramenta 2D no AutoCAD. O agrupamento de serviços com maior impacto no orçamento, a Supraestrutura, foi analisado. Verificou-se que o subgrupo laje de forro teve a maior dispersão e o concreto armado o maior impacto no custo.
\end{abstract}

Palavras-chave: Orçamento; Quantificação; BIM;

\begin{abstract}
Building Information Modelling (BIM) technology presents itself as a tool that allows connection and integration in a way not known before in civil construction, allowing project disciplines to be analyzed in an integrated way and enabling instant improvements. In this study, a comparative analysis of the amounts obtained via the conventional method and via BIM technology was carried out in order to identify the differences between the tools and their impact on the budget. With this objective, a small enterprise was modeled in Revit software and the quantities obtained were compared with those extracted via 2D tool in AutoCAD. The grouping of services with the greatest impact on the budget, the superstructure, was analyzed. It was found that the ceiling slab subgroup had the greatest dispersion and reinforced concrete the greatest impact on cost.
\end{abstract}

Keywords: Budget; Quantification; BIM;

\footnotetext{
${ }^{1}$ Universidade Federal de Minas Gerais (UFMG)

*E-mail: gmtrinta@gmail.com
} 


\section{INTRODUÇÃO}

Com o mercado cada vez mais competitivo de um modo geral, bem como o setor da construção civil, faz-se necessário ter uma boa gestão do orçamento de obra para garantir a sobrevivência e destaque de empresas. Assim, torna-se importante estimar de maneira cada vez mais precisa os quantitativos e os custos, a fim de obter valores o mais próximo possível da realidade (VIANA, 2020).

Nesse sentido, a utilização da ferramenta BIM (Building Information Modelling) tem se destacado no cenário atual da Construção Civil, tendo em vista que proporciona inúmeros benefícios, como a visualização 3D da construção, comunicação direta entre os projetistas e a verificação da compatibilidade entre os projetos. Salienta-se a existência de diversos softwares que utilizam a ferramenta BIM e que podem ser utilizados na elaboração e integração dos projetos, bem como no planejamento, cronograma e orçamento da obra com maior eficiência. A obtenção dos quantitativos por meio da ferramenta permite que se tenha uma redução de tempo na execução assim como demanda uma menor intervenção humana com impacto direto na redução de erros (SAKAMORI e SCHEER, 2016).

Portanto, o Building Information Modelling (BIM), objeto deste estudo, viabiliza todo o processo e tem muito a contribuir, pois auxilia o orçamentista na extração dos quantitativos e elaboração do orçamento, garantindo maior agilidade, assertividade e confiabilidade. Além disso, agiliza alterações de projeto no decorrer da obra, permite antecipar as decisões e especificações de projeto e, ainda, possibilita desenvolver modelos com a sequência de atividades a serem executadas juntamente com seus custos e prazos.

Dessa forma, no presente estudo compara-se os quantitativos extraídos por meio de duas ferramentas diferentes (Revit e AutoCAD) para o agrupamento de serviço com maior impacto no orçamento de um edifício residencial de pequeno porte. A partir disso, discorre-se sobre os principais pontos de conflito entre os métodos e os impactos no orçamento, ressaltando os diferenciais da tecnologia BIM. 


\section{REVISÃO BIBLIOGRÁFICA}

Neste item realiza-se uma revisão da literatura sobre a tecnologia BIM e estudos sobre a sua aplicação, principalmente, com relação à extração de quantitativos para orçamento.

\section{Building Information Modelling}

Para Eastman et al. (2014), os modelos virtuais podem ser detalhados e analisados de uma maneira que não é possível com modelos físicos em escala. Os autores acrescentam que o BIM possibilita a integração das ideias incentivando a colaboração além daquela envolvida nos desenhos e, como consequência, redistribui o tempo e esforços gastos pelos projetistas nas diferentes fases do projeto.

Eastman et al. (2014) e Campestrini et al. (2015) mencionam também outros benefícios do seu uso como a interoperabilidade - caracterizada pela facilitação que múltiplos indivíduos e aplicações atuem no projeto -, a colaboração, a redução de erros pelo uso da detecção de interferências, o estudo da viabilidade do empreendimento a partir de modelos aproximados, a compatibilização dos diferentes projetos (como Arquitetura, Estrutural, Hidrossanitário e Elétrico), redução do desperdício, dentre muitos outros.

Campestrini et al. (2015) também fazem menção aos tipos de modelos BIM 3D, 4D, 5D, 6D, nD. Para cada dimensão, há certas informações que podem ser extraídas. De um modelo 3D, por exemplo, consegue-se o quantitativo e especificações de materiais assim como informações sobre a compatibilização espacial do projeto. No BIM 4D, é possível de se obter também questões de prazo, cronograma e visualização de início e término de tarefas. Já no BIM 5D, consegue-se extrair informações atreladas aos custos de serviços, como as curvas $\mathrm{ABC}$, por exemplo. É válido notar que, com o aumento da dimensão de análise do modelo, cresce também o seu nível de complexidade assim como o número de dados que se consegue obter dele. 


\section{Orçamentação}

Goldman (1997), destaca que o orçamento da obra é uma das principais informações que se deseja conhecer quando se deseja avaliar a viabilidade de determinado projeto. Sabe-se que no setor da construção civil o orçamento tem ainda mais relevância por implicar em gastos consideráveis. O estudo de viabilidade é feito por empresas, muitas vezes, apenas com o projeto arquitetônico em fase de anteprojeto e com os complementares - como o estrutural - não iniciados.

Ferreira (2019) e Mattos (2006) apontam que o orçamento é um estudo do custo total ou parcial de um projeto e que esse custo corresponde à soma de todos os gastos necessários para execução do mesmo, não sendo completo caso exclua algum serviço requerido pela obra. Complementam que os orçamentos apresentam um valor aproximado e que, por mais cuidadoso que o orçamentista seja, ele não tem que ser exato, mas deve seguir determinada linha a fim de se ter maior precisão e reduzir a margem de erros. Ele inclui cálculos baseados em dimensões precisas fornecidas no projeto - como a área de pintura - ou em alguma estimativa - como o volume de escavação do solo.

Ainda, Monteiro e Poças Martins (2013 apud PINTO, 2016) afirmam sobre a importância da precisão de um levantamento de quantidades, por exemplo, para que empresas possam se destacar em processos licitatórios e para que possam elaborar propostas competitivas tanto com relação ao financeiro, como também ao prazo para o cliente.

Além disso, todas as ferramentas BIM, segundo Eastman et al. (2014), fornecem recursos para a extração de quantitativos de componentes, áreas e volumes de espaços, quantidades de materiais e que esses dados são adequados para se ter estimativas aproximadas de custo. Ainda, ressalta que a extração das quantidades não substitui o processo de orçamentação em si, mas que os orçamentistas devem considerar o uso da tecnologia BIM para facilitar a trabalhosa tarefa de levantamento de quantitativos e também para poder visualizar e avaliar de forma rápida e otimizar preços.

Eastman et al. (2014) também citam três métodos principais que os orçamentistas podem utilizar no processo de orçamentação, uma vez que nenhuma ferramenta BIM tem 
todas as funcionalidades de uma planilha eletrônica, são eles: exportar quantitativos de objetos da edificação para um software de orçamentação, conectar a ferramenta BIM diretamente ao software de orçamentação, usar uma ferramenta BIM de levantamento de quantitativos.

\section{METODOLOGIA}

A modelagem foi a ferramenta metodológica adotada para o desenvolvimento deste trabalho. Como apoio, foram utilizados o software Revit para a concepção do modelo e o software Microsoft Excel para auxiliar nas análises.

O estudo teve como base uma edificação de pequeno porte (158 metros quadrados $-\mathrm{m}^{2}$ ) concebida em concreto armado, localizada na região Nordeste do estado de Minas Gerais. Foi feito um comparativo de custos do orçamento obtido via Revit BIM e via AutoCAD para o agrupamento com maior impacto no orçamento. Para isso, foram utilizados conceitos do princípio de Pareto (ou princípio 80/20) como critério para tomada de decisão dos maiores impactos e que será detalhado no tópico dos resultados.

Segundo Koch (2015 apud SANTOS et al., 2020) o princípio de Pareto ou 80/20 aponta que há um desequilíbrio entre as causas e os resultados, em que a maior parte tem baixo impacto e a menor parte tem alto impacto, isto é, os resultados são majoritariamente derivados de uma pequena porção das causas.

Na Figura 1 e Figura 2, estão apresentados o primeiro e o segundo pavimentos da edificação, em planta, os quais fazem parte do projeto executivo fornecido pela empresa. 
Figura 1 - Primeiro pavimento em planta no AutoCAD

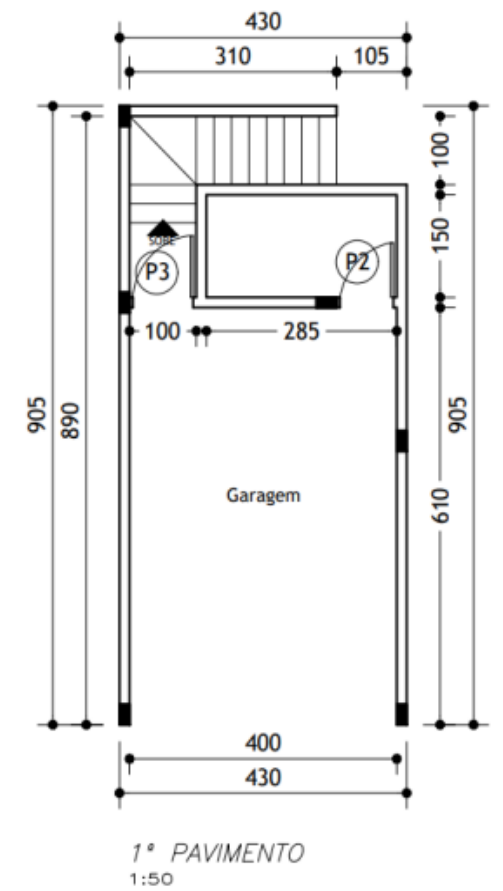

Fonte: Fornecida pela empresa (2020).

Figura 2 - Segundo pavimento em planta no AutoCAD

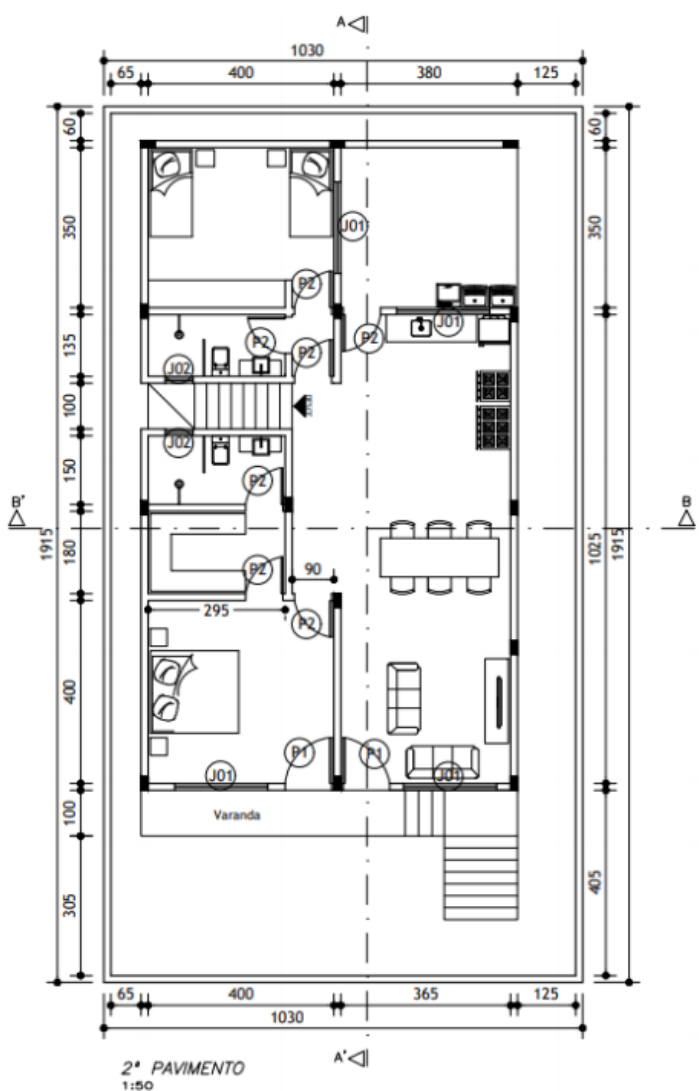

Fonte: Fornecida pela empresa (2020). 


\section{RESULTADOS E DISCUSSÃO}

Foi realizada a modelagem das disciplinas - Arquitetura e Estrutural - por meio de tecnologia BIM com o auxílio do software Revit da Autodesk. A representação da edificação modelada conforme os projetos fornecidos pode ser melhor visualizada na vista 3D na Figura 3.

Figura 3 - Modelagem arquitetônica em perspectiva 3D

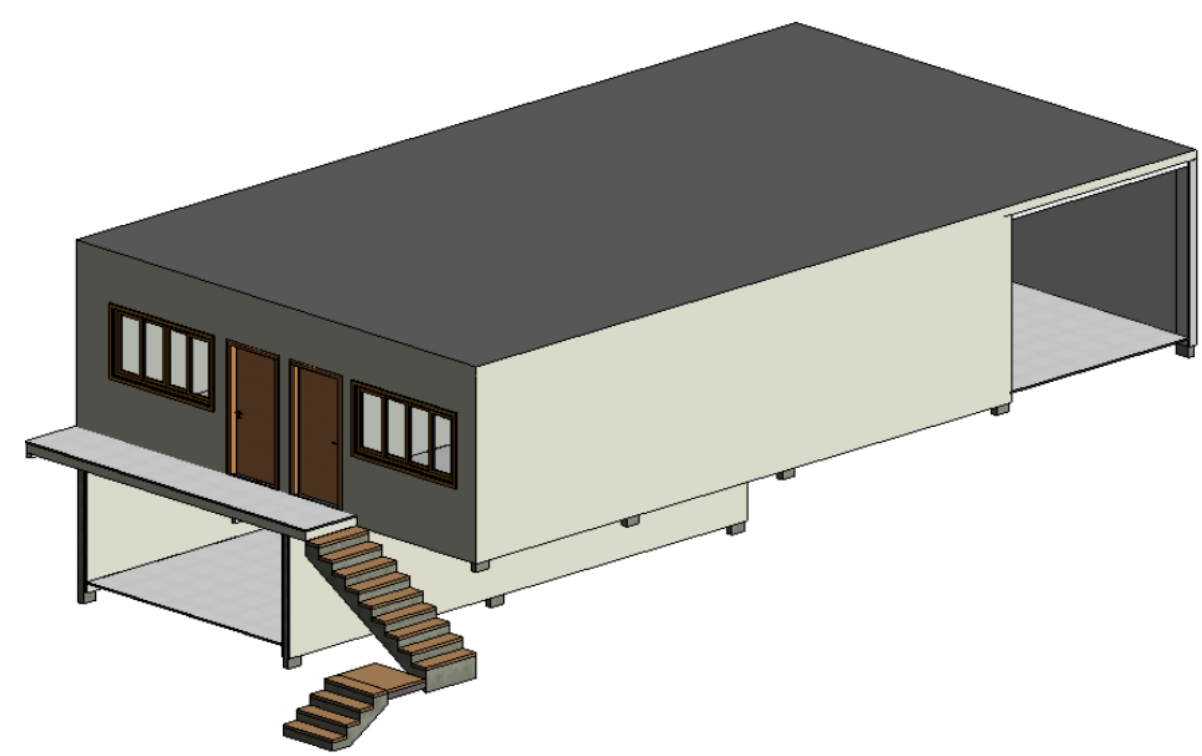

Fonte: Autor.

Para as análises, foi considerado o grupo de serviço com maior impacto no orçamento do projeto feito pela empresa. Para isso, utilizou-se a representação do princípio 80/20 ou princípio de Pareto em que os grupos foram dispostos em ordem de impacto orçamento total, conforme pode ser observado na Tabela 1 e Figura 4. Em destaque na Tabela 1 estão aqueles serviços que representam $80 \%$ do impacto. 
Tabela 1 - Agrupamentos elencados em ordem de impacto

\begin{tabular}{|lccc|}
\hline Agrupamentos & Custo/mil $(\mathbf{R} \$ \mathbf{)}$ & \% do total & Acumulado \\
\hline Supraestrutura & 26,46 & $17,64 \%$ & $17,64 \%$ \\
Revestimentos & 26,17 & $17,45 \%$ & $35,09 \%$ \\
Hidro e elétrica & 18,47 & $12,31 \%$ & $47,40 \%$ \\
Pisos & 14,86 & $9,91 \%$ & $57,31 \%$ \\
Alvenarias & 13,49 & $8,99 \%$ & $66,30 \%$ \\
Esquadrias & 11,29 & $7,52 \%$ & $73,82 \%$ \\
Infraestrutura & 10,65 & $7,10 \%$ & $80,92 \%$ \\
\hline Coberturas & 7,69 & $5,12 \%$ & $86,05 \%$ \\
Louças & 6,27 & $4,18 \%$ & $90,22 \%$ \\
Serv. preliminares & 5,50 & $3,67 \%$ & $93,89 \%$ \\
Outros & 4,16 & $2,77 \%$ & $96,66 \%$ \\
Forros & 3,15 & $2,10 \%$ & $98,76 \%$ \\
Acabamentos & 1,86 & $1,24 \%$ & $100,00 \%$ \\
\hline
\end{tabular}

Fonte: Autor.

Figura 4 - Diagrama de Pareto dos maiores impactos

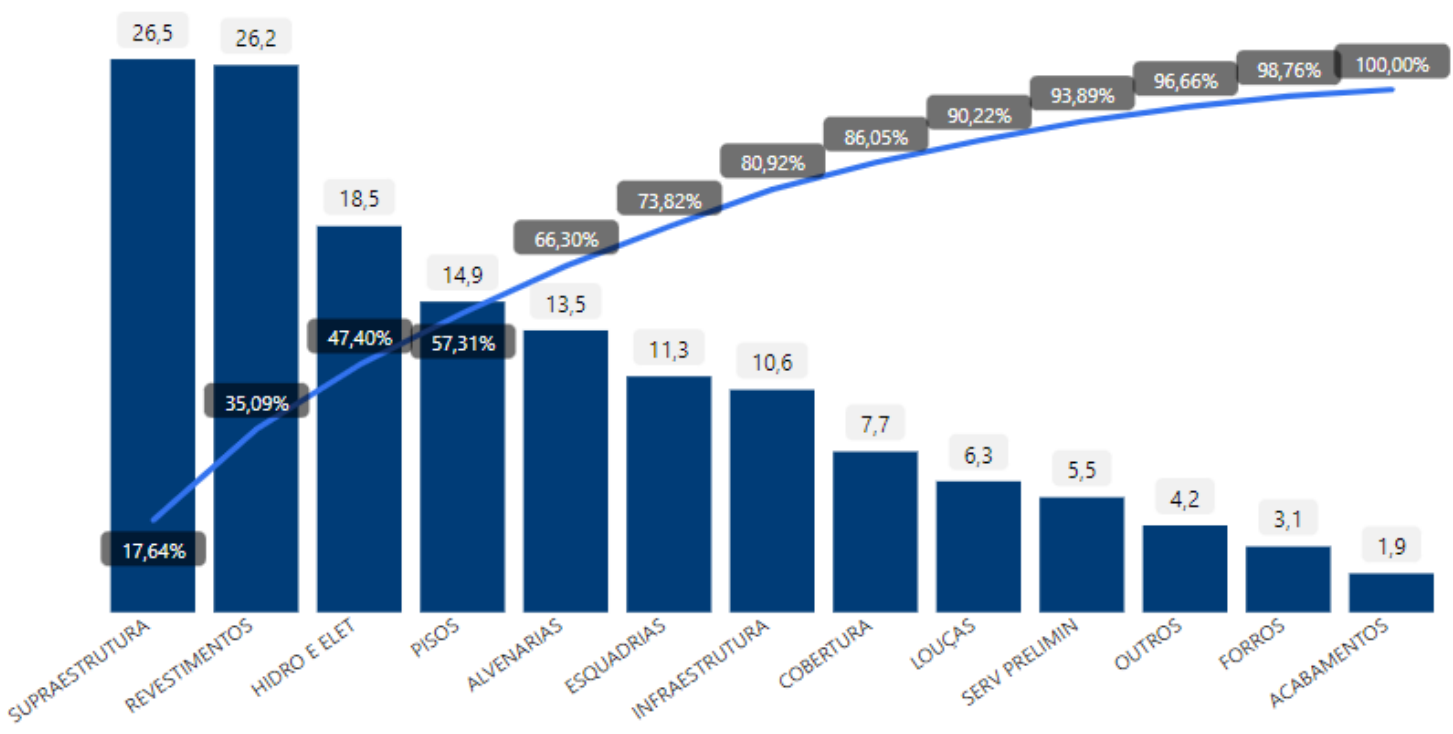

Fonte: Autor.

A análise de comparação dos quantitativos extraídos via AutoCAD e Revit para o agrupamento com maior impacto pelo diagrama - a Supraestrutura - será apresentada no tópico a seguir.

\section{Supraestrutura}

O agrupamento da Supraestrutura tem como subgrupos o volume de concreto $\left(\mathrm{m}^{3}\right)$ e a área das lajes de forro e piso $\left(\mathrm{m}^{2}\right)$. 
A Tabela 2, a Figura 5 e a Figura 6 sintetizam o resultado da análise. Conforme os valores da Figura 5, a laje de forro teve a maior dispersão percentual com uma redução de 10,89\% no quantitativo extraído do Revit com relação ao obtido pelo AutoCAD, já a menor dispersão foi encontrada para a laje de piso, sendo um acréscimo de $0,15 \%$.

Em questões de impacto no orçamento (em R\$), com base na Tabela 2, o concreto armado apresentou a maior diferença, sendo que o valor obtido no Revit foi $\mathrm{R} \$ 1.158,39$ menor que o obtido via AutoCAD, para a Laje de forro, essa diferença também foi de redução com relação ao AutoCAD num total de $\mathrm{R} \$ 744,65$ já para a Laje de piso, houve uma diferença positiva com acréscimo de $\mathrm{R} \$ 3,47$ no custo.

O concreto armado, apesar de não ter a maior dispersão, representou mais de $60 \%$ do impacto, em módulo, da soma total das divergências referentes à Supraestrutura, isto é, da soma total dos valores das dispersões, a maior parcela cabe ao concreto armado, como pode ser observado também na Figura 6.

Tabela 2 - Desvios de quantitativos para a Supraestrutura

\begin{tabular}{lcccc} 
& Qtde CAD & Qtde Revit & $\begin{array}{c}\text { Custo CAD } \\
(\mathbf{R} \$)\end{array}$ & $\begin{array}{c}\text { Custo Revit } \\
(\mathbf{R} \$)\end{array}$ \\
\hline 1. Supraestrutura & & & & \\
\hline 1.1 Concreto armado $\left(\mathrm{m}^{3}\right)$ & 27,45 & 25,62 & $17.375,85$ & $16.217,46$ \\
1.2 Laje de forro $\left(\mathrm{m}^{2}\right)$ & 128,96 & 114,91 & $6.834,88$ & $6.090,23$ \\
1.3 Laje de piso $\left(\mathrm{m}^{2}\right)$ & 38,91 & 38,97 & $2.249,00$ & $2.252,47$ \\
\hline
\end{tabular}

Fonte: Autor.

Figura 5 - Divergência entre as ferramentas para a Supraestrutura

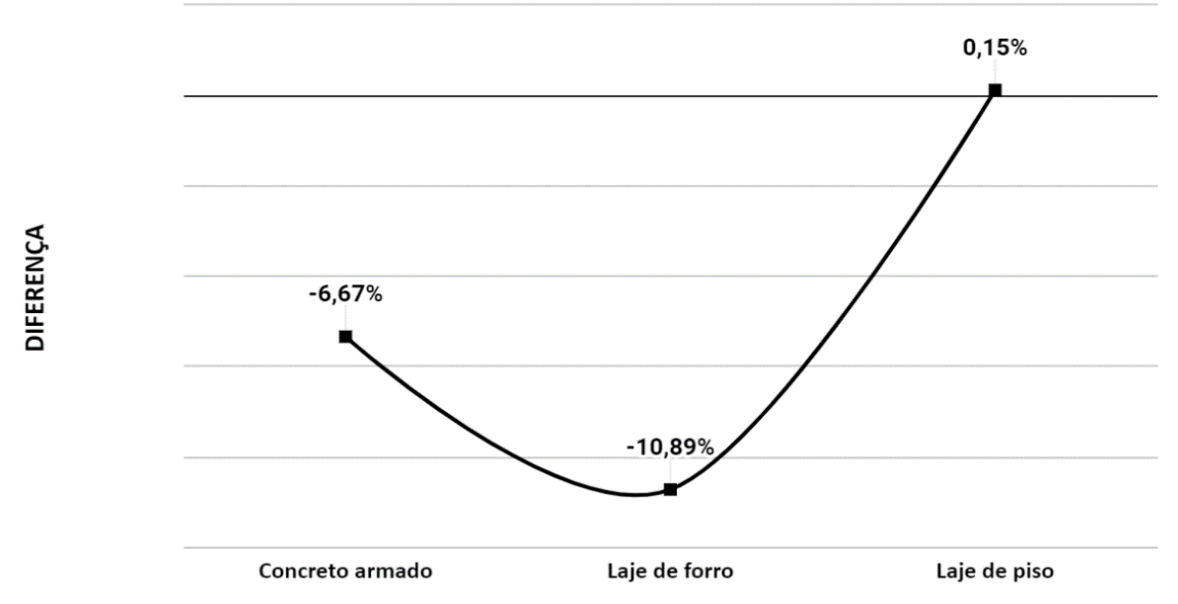

Fonte: Autor. 
Figura 6 - Impacto das divergências no custo para a Supraestrutura

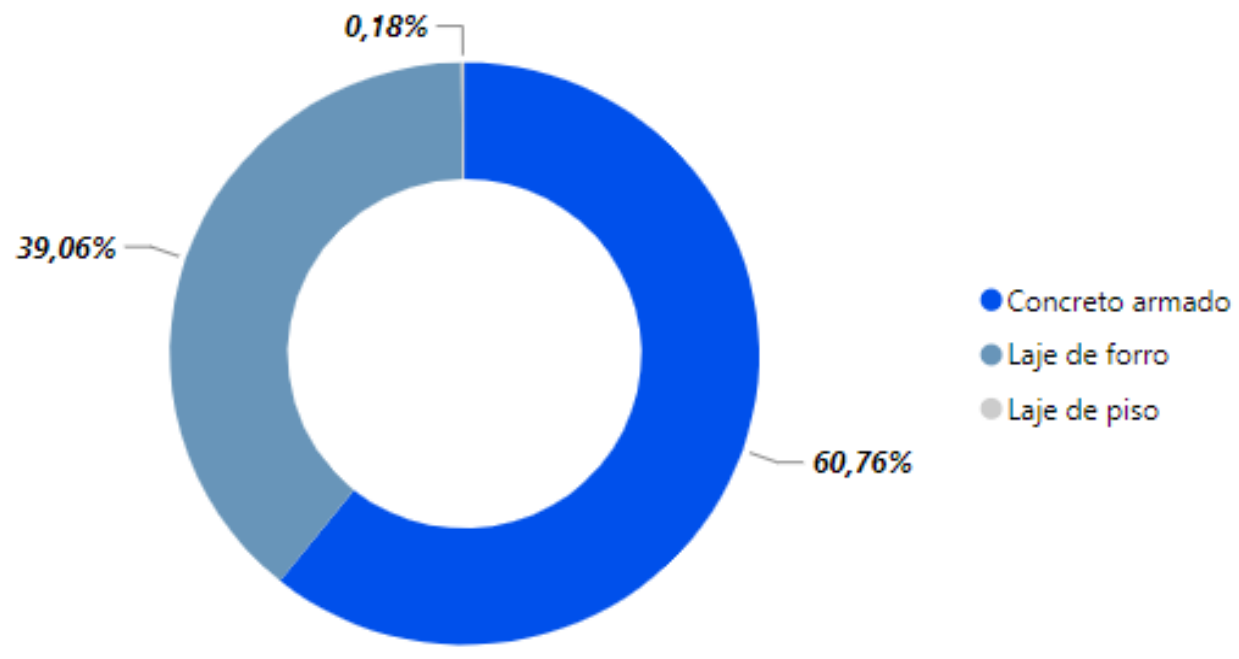

Fonte: Autor.

É válido sublinhar que para as análises feitas neste estudo, considerou-se o impacto no custo em módulo, isto é, não se considerou os valores como negativos e positivos ou como redução e acréscimo, pois tomou-se como cenário ideal a não existência da dispersão seja ela positiva ou negativa.

Além disso, utilizando a formatação "escala de cores" do software Excel, foi elaborada a Tabela 3 em que as cores de cada célula das tabelas se distribuem de acordo com os dados, neste caso, as células com menores valores apresentam uma cor mais clara sendo que as células em cor mais escura representam os maiores valores. A intenção foi avaliar em conjunto as variáveis - dispersão e impacto no custo.

Como pode-se observar na Tabela 3 o agrupamento da Supraestrutura tem como subgrupo com a maior dispersão a laje de forro, no entanto, o maior impacto nos custos é devido ao concreto armado. Não há um subgrupo que se destacou em ambas as categorias ao mesmo tempo, mas é válido sublinhar o concreto armado que teve uma dispersão relativamente pequena $(6,67 \%)$, mas representou um impacto no custo de mais de $60 \%$ dentro do agrupamento, isso devido ao seu maior custo unitário. 
Tabela 3 - Desvios de quantitativos para a Supraestrutura

\begin{tabular}{lcc}
\hline & $\begin{array}{c}\text { Diferença entre } \\
\text { ferramentas }\end{array}$ & Impacto no custo \\
\hline Concreto armado & $6,67 \%$ & $60,76 \%$ \\
Laje de forro & $10,89 \%$ & $39,06 \%$ \\
Laje de piso & $0,15 \%$ & $0,18 \%$ \\
\hline
\end{tabular}

Fonte: Autor.

Considerando-se a diferença entre a soma dos custos totais obtidos via AutoCAD com os extraídos pelo Revit para a Supraestrutura, tem-se uma diferença no custo de $\mathrm{R} \$ 1.899,57$ que representa uma economia de cerca de 7,18\%, como pode ser observado na Tabela 4

Tabela 4 - Diferença geral para a Supraestrutura

\begin{tabular}{lcc}
\hline & Custo CAD $(\mathbf{R} \mathbf{\$})$ & Custo Revit $(\mathbf{R} \mathbf{)})$ \\
\hline Custo total & $26.459,73$ & $24.560,16$ \\
Diferença & & $-1.899,57$ \\
\hline
\end{tabular}

Fonte: Autor.

Análise global

Além da análise específica para o agrupamento da Supraestrutura, foi feita também uma análise global das dispersões e impactos no custo a fim de observar o comportamento dos demais agrupamentos frente à Supraestrutura. Para isso, foi calculada a dispersão global de cada grupo com base nas dispersões dos subgrupos componentes, por exemplo, para a Supraestrutura foi feita a média dos custos devido às dispersões dos subgrupos (concreto, lajes de piso e de forro) e em seguida comparada essa média com a dos demais agrupamentos. Ainda, seguiu-se o mesmo raciocínio para identificar o impacto do custo referente aos agrupamentos. Para melhor visualizar e comparar as dispersões e os impactos no custo de forma global, foram desenvolvidas a Figura 7 e a Figura 8.

Dentre os agrupamentos, os Revestimentos apresentaram a maior dispersão média (redução de 19,10\%), seguido dos Pisos (redução de 13,27\%) e pela Supraestrutura sendo que a menor delas ocorreu nas Alvenarias, como pode ser visto na Figura 7. Pela Figura 8 nota-se que o maior impacto no custo devido às dispersões é também devido ao grupo dos Revestimentos com pouco mais de $38 \%$. 
É válido destacar que que todos os grupos apresentaram dispersão média de redução nas quantidades obtidas via BIM se comparada às obtidas pelo 2D (Figura 7).

Figura 7 - Divergência entre as ferramentas de forma global para os agrupamentos

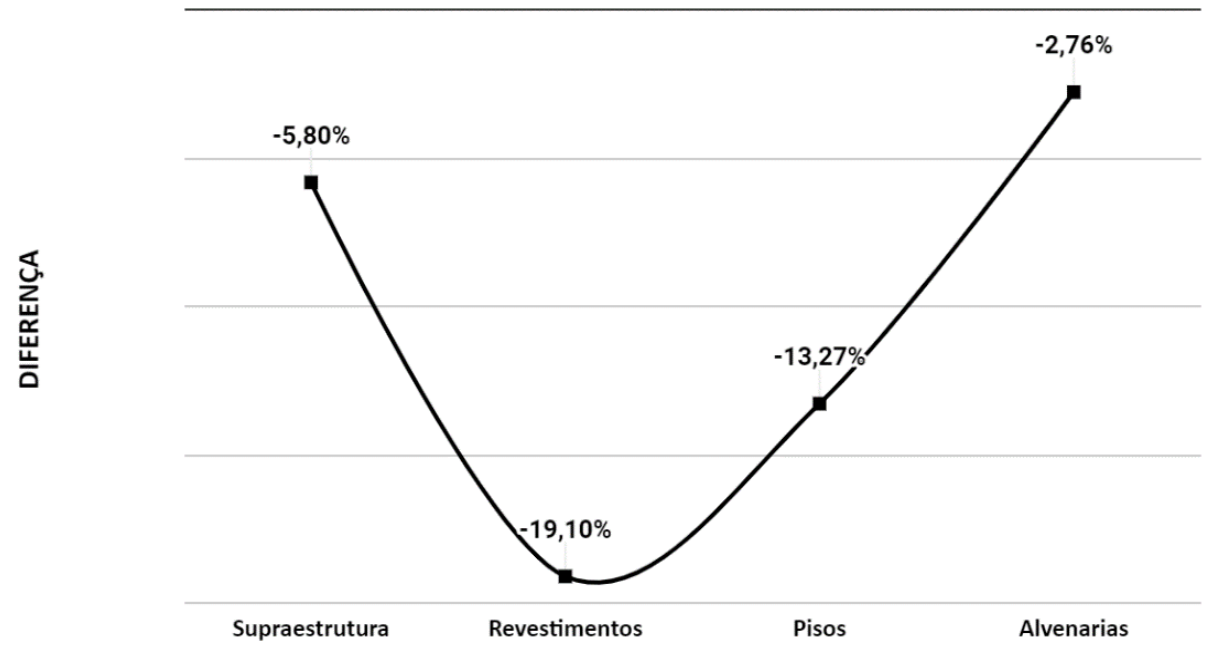

Fonte: Autor.

Figura 8 - Impacto das divergências no custo de forma global para os agrupamentos

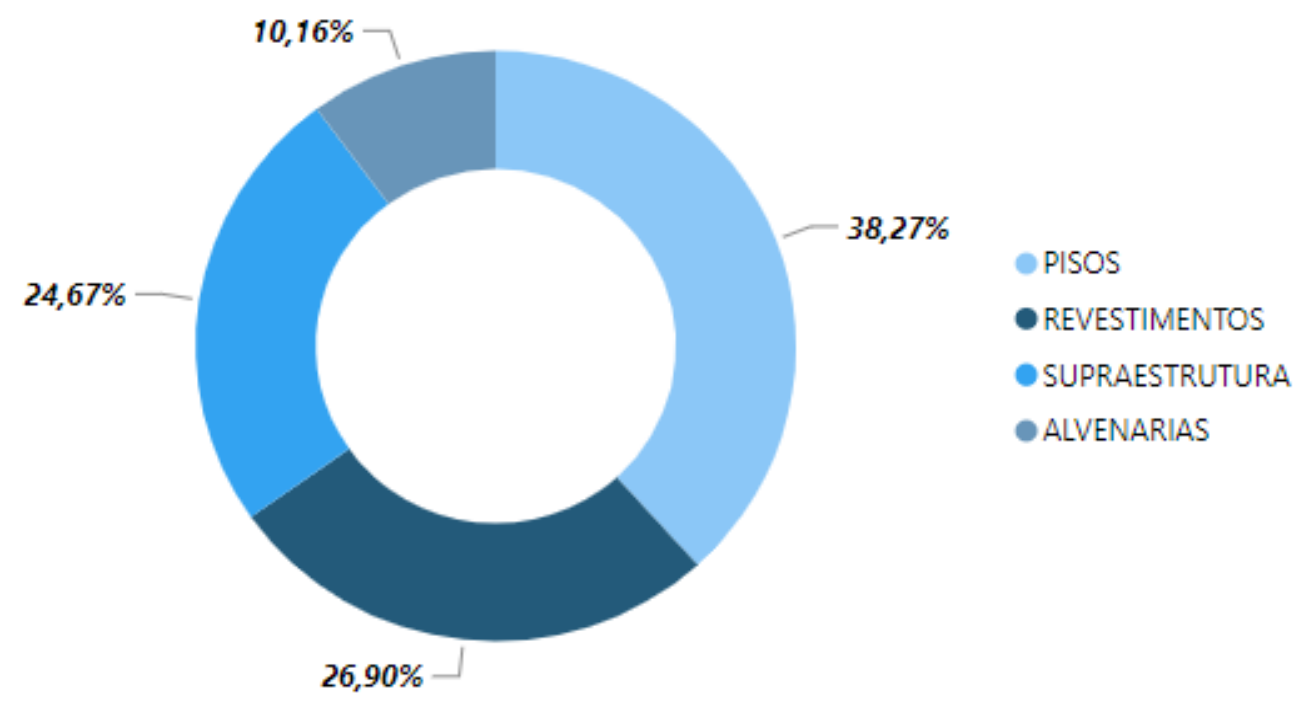

Fonte: Autor. 


\section{CONSIDERAÇÕES FINAIS}

Este trabalho teve como objetivo principal a análise comparativa entre a extração de quantitativos de um projeto de uma obra de pequeno porte por meio de duas ferramentas diferentes, em que se pretendia identificar possíveis divergências nos resultados para o agrupamento de serviço com maior impacto no orçamento, a Supraestrura.

Diante das análises dos resultados realizada, percebeu-se que houve uma diferença considerável entre os quantitativos obtidos pelo método convencional - via ferramenta 2D no AutoCAD - quando comparados aos obtidos por meio do software Revit com destaque para a Laje de Forro com 10,89\% de divergência e para o concreto armado representando mais de $60 \%$ de impacto no custo dentro do agrupamento.

É válido sublinhar a superioridade da tecnologia BIM frente ao 2D, seja na automatização do processo de extração de quantitativos, na confiabilidade para obtenção de orçamentos mais assertivos e também na facilitação e permissão da visualização em vários ângulos do projeto 3D durante o processo de modelagem, como citado por Protázio e Rêgo (2012).

Ainda, ter maior precisão no processo de extração de quantitativos e orçamentação pode garantir o destaque necessário para uma dada empresa elaborar uma proposta mais competitiva e se diferenciar. Este é apenas um ponto, dentre vários outros positivos que menores divergências em orçamentos podem implicar.

Como forma de consolidar os resultados obtidos, é interessante que haja um novo estudo com uma obra maior, com um orçamento e agrupamentos mais detalhados. Além disso, complementar o estudo com a continuação da modelagem para além do BIM 3D e verificar os impactos de cada agrupamentos e subgrupos em outras dimensões, como por exemplo, o tempo. 


\section{REFERÊNCIAS}

CAMPESTRINI, T. F. et al. Entendendo BIM. Curitiba, Paraná, Brasil: 2015.

EASTMAN, C.; TEICHOLZ, P.; SACKS, R.; LISTON, K. Manual de BIM: Um Guia de Modelagem da Informação da Construção para Arquittos, Engenheiros, Gerentes, Construtores e Incorporadores. Porto Alegre: Bookman, 2014. 483p.

FERREIRA, D. Planejamento e orçamento de obra: roteiro e estudo de caso de elaboração de um planejamento e orçamento de obras. 2019. Monografia (Especialização em Materiais de Construção) - Escola de Engenharia, Universidade Federal de Minas Gerais, Belo Horizonte, 2019.

GOLDMAN, P. Introdução ao planejamento e controle de custos da construção civil. 3ed. São Paulo: Pini, 1997. ISBN 85-7266-072-0

KOCH, R. O Poder 80/20: Os segredos para conseguir mais com menos nos negócios e na vida. São Paulo: Gutenberg, 2015 apud SANTOS, A. P. et al. Utilização da ferramenta Diagrama de Pareto para auxiliar na identificação dos principais problemas nas empresas, 2020, Araçatuba, SP: UniSalesiano, 2020, p. 1-12.

MATTOS, A. D. Como preparar orçamentos de obras. 1. ed. [s.1.] Editora PINI Ltda., 2006.

MONTEIRO, A.; POÇAS MARTINS, J. A survey on modeling guide lines for quantity takeoff-oriented BIM-based design. Automation in Construction, Amsterdam v.35, p. 238- 153, 2013. ISSN 09265805 (ISSN). Disponível em: https://www.scopus.com/record/display.uri?eid=2-s2.084884498519\&origin=inward\&txGid=5907bee79758e368385bc798b7833369 apud PINTO, A. C. B. C. Propostas técnicas para obras de edificações: estudos de caso. 2016. 124p. (Mestrado). Programa de pós graduação em engenharia civil, Universidade de São Paulo, São Paulo.

PINTO, A. C. B. C. Propostas técnicas para obras de edificações: estudos de caso. 2016. 124p. (Mestrado). Programa de pós graduação em engenharia civil, Universidade de São Paulo, São Paulo.

PROTÁZIO, J. V. B.; RÊGO, R.M. Estudo e avaliação de tecnologias bim para projetação em arquitetura, engenharia e construção. IFPE, Recife/PE, dezembro, 2012.

SAKAMORI, M. M.; SCHEER, S. Processo de extração de quantitativos de um modelo BIM 5D. In: Congresso técnico científico da engenharia e da agronomia. Foz do Iguaçu, 2016. Disponível em: encurtador.com.br/fuGLP.Acesso em: 30 maio 2021.

SANTOS, A. P. et al. Utilização da ferramenta Diagrama de Pareto para auxiliar na identificação dos principais problemas nas empresas, 2020, Araçatuba, SP: UniSalesiano, 2020, p. 1-12. 
VIANA, L. Comparativo de custos dos sistemas construtivos wood frame e concreto armado para edifício utilizando BIM 5D. 2020. Dissertação (Mestrado em

Construção Civil) - Escola de Engenharia, Universidade Federal de Minas Gerais, Belo Horizonte, 2020.

Recebido em: 03/01/2022

Aprovado em: 30/01/2022

Publicado em: 03/02/2022 